\title{
20

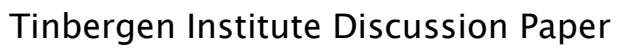 \\ Value of Time by Time of Day: \\ A Stated-Preference Study
}

Yin-Yen Tseng

Erik Verhoef

Dept. of Spatial Economics, VU University Amsterdam, and Tinbergen Institute. 


\section{Tinbergen Institute}

The Tinbergen Institute is the institute for economic research of the Erasmus Universiteit Rotterdam, Universiteit van Amsterdam, and Vrije Universiteit Amsterdam.

Tinbergen Institute Amsterdam

Roetersstraat 31

1018 WB Amsterdam

The Netherlands

Tel.: $\quad+31(0) 205513500$

Fax: $\quad+31(0) 205513555$

Tinbergen Institute Rotterdam

Burg. Oudlaan 50

3062 PA Rotterdam

The Netherlands

Tel.: $\quad+31(0) 104088900$

Fax: $\quad+31(0) 104089031$

Most TI discussion papers can be downloaded at http:/ /www.tinbergen.nl. 


\title{
VALUe OF TIME BY TIME OF DAY
}

\section{A Stated-Preference Study ${ }^{\#}$}

\author{
Yin-Yen Tseng $^{*}$ and Erik T. Verhoef** \\ Department of Spatial Economics \\ VU University Amsterdam \\ De Boelelaan 1105 \\ 1081 HV Amsterdam, The Netherlands \\ Phone: +31-20-4446098 \\ Fax: +31-20-4446004 \\ Email: ytseng@feweb.vu.nl / everhoef@feweb.vu.nl
}

This version: 07/11/07

Key words: Value of Travel Time Savings, Value of Schedule Delay, Value of Unreliability, Stated Choice

JEL codes: R41, D12

\begin{abstract}
This paper proposes an alternative, dynamic framework for estimating time-varying values of travel time savings and values of schedule delay, in which time-preferences are represented as the time-varying excess willingness to pay (EWPT) to being in the one location, over being elsewhere. It is shown how the conventional linear model, with time-independent values of travel time savings and schedule delay costs, is a special case of our model, and that it is implausible particularly in that it implicitly assumes that the willingness to pay for spending a minute at home instead of being in the vehicle does not vary by time of day, even not for very early departures. The framework is applied to SP data representing the respondents' departure time choices for the morning commute. The results suggest that individuals' timerelated shadow prices indeed vary strongly over the morning peak, and values of travel time savings are consequently strongly time-dependent, following plausible and intuitive patterns.
\end{abstract}

\footnotetext{
\# This research was partly financed through the NWO/Connekt VEV project on "A Multidisciplinay Study of Pricing Policies in Transport - An Economic Perspective"; nr. 014-34-351. Financial support is gratefully acknowledged. We thank the MD-PIT team, and Dirk van Amelsfort and Barry Ubbels in particular, for their efforts in collecting the data used in this study. Very helpful comments by two reviewers and Kenneth Small are also gratefully acknowledged. All remaining errors are ours alone.

* Corresponding author.

**Affiliated to the Tinbergen Institute, Roetersstraat 31, 1018 WB Amsterdam.
} 


\section{Introduction}

The value of travel time savings (VTTS), often abbreviated as 'value of time' (VOT), is of central interest in transport research. The VTTS is often among the bigger benefit components in the assessment of transport investments. It is also an important parameter in the analysis of travel behaviour and in traffic assignment models. Becker (1965) was probably the first to introduce the allocation of time over various activities in the analysis of consumer behaviour, thus offering the micro-economic framework needed to establish the shadow price of time savings. Further contributions from Johnson (1966) who introduced work time in the utility function, Oort (1969) who did the same for travel time, and DeSerpa (1971) who added technical constraints, showed that the VOT at various activities does not need to be equal to the wage rate - justifying further research into the empirical estimation of VTTS. In such research, the VTTS is usually derived as the marginal rate of substitution between travel time and cost coefficients, typically as found in discrete choice models of stated preference data, revealed preference data, or a combination of these (e.g. Small, Winston and Yan, 2005). This ratio is exactly the VTTS in DeSerpa's (1971) framework (Bates, 1987).

An important addition to this framework was made by Small (1982), who explicitly included the scheduling of activities - the morning commute in particular - in the analysis. Inspired by the work of Vickrey (1969) on the dynamic equilibrium and optimum for queuing behind a bottleneck, Small allowed for disutility from early or late arrivals at work. With a simple linear utility specification, this leads to three relevant time-related shadow prices: a value of travel delay which is usually denoted $\alpha$ in the relevant literature (e.g., Arnott, De Palma and Lindsey, 1993), a shadow price of arriving early $(\beta)$, and a shadow price of late arrivals ( $\gamma$ ). Small's (1982) model has become the workhorse model to incorporate within-theday dynamics in the valuation of travel time components.

A particular aspect of Small's basic linear model is that the inconvenience of an early trip is attributed to an early arrival at work. This implies, in terms of the notation just introduced, that a constraint $\beta<\alpha$ should be imposed - unless one is willing to accept that an individual may prefer to stay in the parked car when arriving early, over getting out of the car and into the office (or factory). Moreover, $\beta>\alpha$ also implies that for a deterministic dynamic equilibrium with homogeneous travellers, a person who arrives after another person should have departed before that other person for total trip cost to be constant by arrival time (which is the natural dynamic equilibrium condition for homogeneous travellers). But consistent overtaking as a structural equilibrium phenomenon is not plausible.

Nevertheless, the inequality $\beta<\alpha$ need not always be satisfied in applied work, especially not for linear specifications. Indeed, the focus on deviations from desired arrival times, in combination with the constancy of shadow prices $\alpha, \beta$ and $\gamma$ for a linear utility function, makes it impossible to capture the intuitive notion that rescheduling to earlier time slots becomes increasingly inconvenient as increasingly more valuable moments spent at home (possibly sleeping) are to be given up. Allowing for this by introducing a non-linear effect of schedule delay early upon utility is somewhat ad hoc: it can partly account for the 
effect mentioned, but leads to the risk of $\beta>\alpha$ for early arrivals and may assign different penalties for early departures from home at the same moment when trip durations vary.

Our purpose is to propose a variant of Small's model that explicitly allows for nonlinear effects. We present a model that estimates two time-dependent utility components, namely the per-unit-of-time willingness to pay for being at home over being in the car $H(t)$ (a negative value would imply a preference for the car), and the per-unit-of-time willingness to pay for being at work over being in the car $W(t)$. We show how the implied two functions in fact define three time-dependent functions $\alpha(t), \beta(t)$ and $\chi(t)$, which are the time-varying equivalents of the conventional shadow prices $\alpha, \beta$ and $\gamma$. A recent study of Liu, He and Recker (2007) provides empirical evidence that the values of time and unreliability vary over the peak. Our approach differs from theirs in at least two respects: first, we explicitly include scheduling considerations in the model; and secondly, our approach allows an individual's value of time to vary over time, whereas the empirical time-dependency of the value of time in the analysis of Liu et al. (2007) may equally well result from different departure time choices from different travellers with different values of time and unreliability.

Section 2 present our framework and discusses how it has the conventional linearized version of Small's (1982) model as a special, restricted case. Section 3 introduces the empirical application of our model, and Section 4 presents estimation results. Section 5 concludes.

\section{Theoretical framework}

Consider an individual who has to decide whether or not to travel to work in the morning, and if so, at what time. We hypothesize that this individual's utility over the full morning period (that is, a period between two instants $t_{b}$ ("begin") and $t_{e}$ ("end"), chosen such that it is long enough to encompass all possibly relevant departure times from home and arrival times at work) can be found by integrating, over the relevant periods, particular functions that represent the per-unit-of-time utility of being at a certain place. To simplify matters, let us assume that the individual considers three possible places where he can be: at home, in the vehicle, or at work (all other possible places are considered inferior to even the least preferable of these three at any relevant instant $t$ ). Let every unit of time spent at home produce a time-varying utility $h(t)$, and define analogously the utility in the vehicle $v(t)$, and at work $w(t)$. Assume that each of these functions is continuous and smooth.

We ignore complexities that may stem from the dependence of the marginal utility of income upon the scheduling decisions in the morning period, and assume it is constant. We can then choose units of utility such that differences between any pair from the triplet $\{h(t)$, $v(t), w(t)\}$ denote the individual's willingness to pay to spend, at time $t$, one unit of time at the more preferred location rather than the less preferred one.

Indeed, because we only consider three possible locations where the individual can be, behaviour will be determined entirely by differences in utility levels. We can therefore simplify notation by equating one of the three utility levels to 0 throughout the period $\left[t_{b}, t_{e}\right]$, which only means that calculated utility levels are reduced by a constant equal to the integral 
of this reference utility function between $t_{b}$ and $t_{e}$. We choose $v(t)$ as the reference, so that we are left with two functions that we define as follows:

$$
\begin{aligned}
H(t) & =h(t)-v(t) \\
W(t) & =w(t)-v(t)
\end{aligned} .
$$

$H(t)$ therefore gives the willingness to pay to spend a unit of time at home rather than in the vehicle at time $t$, and $W(t)$ does the same for work versus the vehicle. We will refer to the functions $H(t)$ and $W(t)$ as excess-willingness-to-pay functions, EWTP-functions in brief. Note that there is a priori reason for restricting either EWTP to be positive; a negative value would be consistent with findings reported by, inter alios, Redmond and Mokhtarian (2001), on how travellers may sometimes attach a positive utility to extra time spent in the car.

Figure 1 provides an example, where we made the plausible assumptions that the individual finds it especially at early hours attractive to spend time at home rather than in the vehicle, while the attractiveness of being at work increases rapidly within a relatively short time-span around the official work start time, and remains rather flat both before and after that moment.

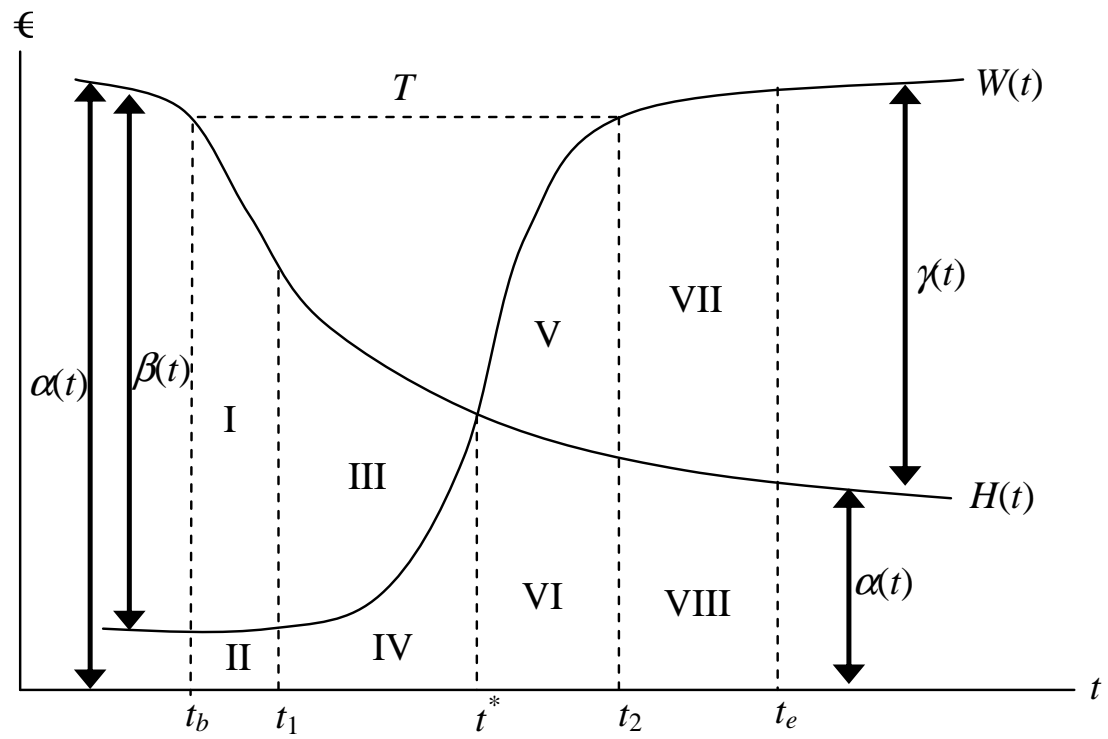

Figure 1. The EWTP-functions for being at home $(H(T))$ and at work $(W(T))$

Let us now define $t_{D}$ and $t_{A}$ as the moments of departure and arrival. If the trip from home to work would require no travel time, so that $t_{A}=t_{D}$, the individual would travel at the instant that $H(t)$ and $W(t)$ intersect, at $t^{*}$. It is tempting to call $t^{*}$ the "desired arrival time", but it actually is most desirable only when travel would take no time. With a given trip duration $T$, the individual would otherwise prefer to depart and arrive at moments $t_{D}$ and $t_{A}$ such that $H\left(t_{D}\right)$ and $W\left(t_{A}\right)$ are equalized while $t_{A}-t_{D}=T$ is satisfied. The trip between $t_{b}$ and $t_{2}$ in Figure 
1 is an example of such an optimally timed trip, for which $t_{2}$ is the desired arrival time. ${ }^{1}$ Therefore, because $t^{*}$ is the desired arrival time only when travel time is zero, we will refer to $t^{*}$ as the "ideal arrival time".

It seems natural to define the individual's travel cost $c\left(t_{D}, t_{A}\right)$ such that is zero for an optimally timed zero-duration trip: $c\left(t^{*}, t^{*}\right)=0$. The willingness to pay for being able to make this trip, over the worst possible situation of being in the vehicle between instants $t_{b}$ and $t_{e}$, is given by the sum of all areas I - VIII in Figure 1. Travel cost $c(\cdot)$ for other trips can be identified graphically in Figure 1 as areas below the maximum of $H(t)$ and $W(t)$ when driving, and between $H(t)$ and $W(t)$ between moments $t_{A}$ and $t^{*}$ when arriving before $t^{*}$, and between $t^{*}$ and $t_{D}$ when departing after $t^{*}$. These areas together namely give the willingness to pay for making the ideal optimally timed zero-duration trip, over the trip under consideration.

Another way to identify the same areas for a given trip is to take the area below $H(t)$ when driving (i.e., between $t_{D}$ and $t_{A}$ ), and add to it the area between $H(t)$ and $W(t)$ between moments $t_{A}$ and $t^{*}$ when arriving before $t^{*}$, or between $t^{*}$ and $t_{A}$ when departing after $t^{*}:^{2}$

$c\left(t_{D}, t_{A}\right)=\int_{t_{D}}^{t_{A}} H(t) \mathrm{d} t+\left\{\begin{array}{ll}\int_{t_{A}^{*}}^{t^{*}}(H(t)-W(t)) \mathrm{d} t \quad \text { if } \quad t_{A}<t^{*} \\ \int_{t_{A}^{*}}(W(t)-H(t)) \mathrm{d} t \quad \text { if } \quad t_{A} \geq t^{*}\end{array}\right.$.

This can be verified by checking the following travel costs for three different types of trips in Figure 1, namely one with both the departure and arrival before $t^{*}$, one with a departure before $t^{*}$ and an arrival after $t^{*}$, and one with departure and arrival both after $t^{*}$ :

$$
\begin{aligned}
& c\left(t_{b}, t_{1}\right)=\mathrm{I}+\mathrm{II}+\mathrm{III} \\
& c\left(t_{1}, t_{2}\right)=\mathrm{III}+\mathrm{IV}+\mathrm{V}+\mathrm{VI} . \\
& c\left(t_{2}, t_{e}\right)=\mathrm{V}+\mathrm{VII}+\mathrm{VIII}
\end{aligned}
$$

It is now convenient to define the following functions:

\footnotetext{
${ }^{1}$ With time-varying travel times, equalization of $H\left(t_{D}\right)$ and $W\left(t_{A}\right)$ is of course no longer the appropriate necessary equilibrium condition. The optimality condition for such cases is straightforward to express after specifying a travel time function $T\left(t_{D}\right)$ and replacing $t_{A}$ by $t_{D}+T\left(t_{D}\right)$. It next involves minimization of equation (2) below with respect to $t_{D}$. This leads to the equilibrium condition: $H\left(t_{D}\right)=\left[1+\mathrm{d} T\left(t_{D}\right) / \mathrm{d} t_{D}\right] \cdot W\left(t_{A}\right)$. Note how it has the constant travel time case discussed in the main text as a special case, where the second term in the square bracket is zero. Otherwise, it corrects for changes in travel time where the departure time is marginally adjusted. With timevarying travel times, an arrival before $t^{*}$ or a departure after $t^{*}$ may occur in equilibrium.

${ }^{2}$ Equivalently, we could specify the model such that $W(t)$ becomes the time-varying value of travel time. This involves schedule delay terms that are the integral of $[H(t)-W(t)]$ between $t_{D}$ and $t^{*}$ for departures before $t^{*}$, and the integral of $[\mathrm{W}(t)-H(t)]$ between $t^{*}$ and $t_{D}$ for departures after $t^{*}$. The fact that we can do this is consistent with our model being symmetric between $H(t)$ and $W(t)$. The specification in the main text has the obvious advantage of being directly comparable to the conventional model. The alternative described in this footnote, which relates schedule delay costs to deviations from the ideal departure time, might be more intuitive to describe the afternoon peak.
} 


$$
\begin{aligned}
& \alpha(t)=H(t) \\
& \beta(t)=H(t)-W(t) . \\
& \gamma(t)=W(t)-H(t)
\end{aligned}
$$

We can then rewrite the cost function in (2) as:

$c\left(t_{D}, t_{A}\right)=\int_{t_{D}}^{t_{A}} \alpha(t) \mathrm{d} t+\left\{\begin{array}{ll}\int_{t_{A}^{*}}^{t^{*}} \beta(t) \mathrm{d} t & \text { if } \quad t_{A}<t^{*} \\ t_{A} & \\ \int_{t_{A}^{*}} \gamma(t) \mathrm{d} t \quad \text { if } \quad t_{A} \geq t^{*}\end{array}\right.$.

The arrows in Figure 1 represent these functions $\alpha(t), \beta(t)$ and $\chi(t)$. These functions are helpful in understanding how the conventional linearized version of Small's (1982) model is a special case of our model. The cost function in the conventional linear model can be written as follows:

$c\left(t_{D}, t_{A}\right)=\alpha \cdot\left(t_{A}-t_{D}\right)+\left\{\begin{array}{lll}\beta \cdot\left(t^{*}-t_{A}\right) & \text { if } & t_{A}<t^{*} \\ \gamma \cdot\left(t_{A}-t^{*}\right) & \text { if } & t_{A} \geq t^{*}\end{array}\right.$.

This is of course a special case of the cost function in (5), with the functions $\alpha(t), \beta(t)$ and $\chi(t)$ all constant over time and equal to $\alpha, \beta$ and $\gamma$, respectively. Figure 2 depicts the associated variant of Figure 1.

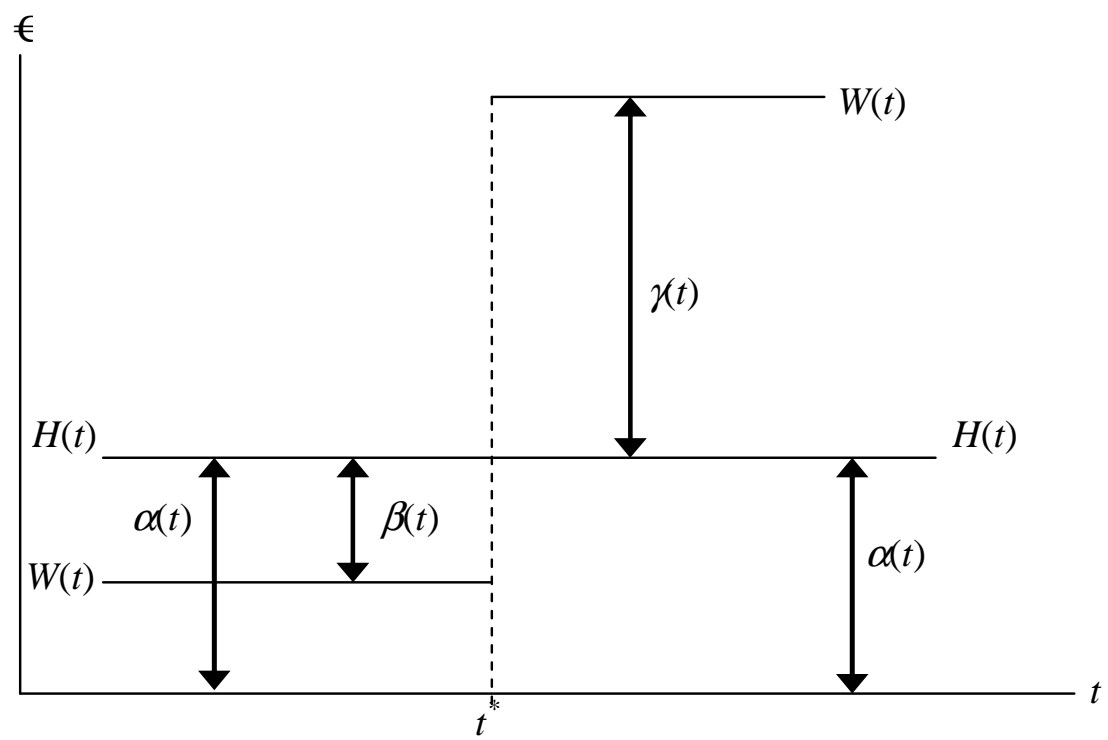

Figure 2. The EWTP-functions in the conventional linearized model

The most striking difference between Figure 1 and 2 is that $H(t)$ is constrained to be constant in the latter, because $\alpha(t)$ is. The inconvenience of an early schedule insofar as affecting the 
time otherwise spent home, perhaps sleeping, can therefore not be properly reflected. A minute spent at home, instead of in the vehicle, is constrained to be equally valuable at 5 a.m. as at 8 a.m. Introspection suggests that this is not plausible, and empirical evidence in the next section will confirm this. The other difference is that $W(t)$ is piecewise constant in Figure 2. Although this is restrictive, too, it seems less so than the first difference, of imposing constancy of $H(t)$ over time.

Finally, it can be noted that the proposed framework is related to recent developments in activity-based modelling (e.g., Ettema and Timmermans, 2003; Ashiru, Polak and Noland, 2004), which also involve the modelling of dynamic scheduling decisions. Our approach is different in that we characterize time-dependent utility functions entirely in terms of timevarying shadow prices, the EWTP-functions, as a dynamic generalization of the conventional constant shadow prices $\alpha, \beta$ and $\gamma$.

\section{Empirical application}

\subsection{Empirical specifications}

The conventional linearized model of equation (6) is easily operationalized for application in a random-utility discrete-choice model through the following specification of a linear systematic utility function:

$V=\beta_{\alpha} \cdot T+\beta_{\tau} \cdot \tau+\left\{\begin{array}{lll}\beta_{\beta} \cdot\left(t^{*}-t_{A}\right) & \text { if } & t_{A}<t^{*} \\ \beta_{\gamma} \cdot\left(t_{A}-t^{*}\right) & \text { if } & t_{A} \geq t^{*}\end{array}\right.$,

with $\tau$ denoting a monetary attribute such as a toll. With the coefficients $\beta$ estimated, one can immediately determine $\alpha=\beta_{d} / \beta_{i} ; \beta=\beta_{\beta} / \beta_{\tau}$; and $\gamma=\beta_{\gamma} / \beta_{\tau}$. With random utility for alternative $j$ for individual $n$ defined as:

$U_{j n}=V(\cdot)+\varepsilon_{j n}$,

conventional multinomial logit or probit discrete-choice models arise under particular assumptions on the distribution of the random terms $\varepsilon_{j n}$.

The operationalization of the more flexible model of (2) and (4) is less straightforward. One option would be to impose functional forms for $H(t)$ and $W(t)$ as functions of time, and to estimate the relevant parameters. Another possibility, pursued below, could be characterized as 'flexible' and divides the morning peak period up in a number of smaller intervals, with $H(t)$ and $W(t)$ constant within an interval but free to vary between them. This has the advantage of not imposing any a priori assumption on the possible time patterns of these functions, but the obvious disadvantage of restricting $H(t)$ and $W(t)$ to be constant within an interval. Because we are interested primarily in detecting the pattern of time variation in $\alpha(t)$ over the peak, if any, we judged the advantage to outweigh the disadvantage.

The systematic utility function can be written after defining $T_{i}$ as the amount of time spent driving during time interval $i, T_{i}^{E}$ as the amount of time spent at work before the ideal 
arrival time $t^{*}$ during time interval $i$, and $T_{i}^{L}$ as the amount of time not (yet) spent at work after $t^{*}$ during time interval $i$. This leads to the following discrete-time version of equation (5):

$$
c\left(t_{D}, t_{A}\right)=\sum_{i} \alpha_{i} \cdot T_{i}\left(t_{D}, t_{A}\right)+\sum_{i} \beta_{i} \cdot T_{i}^{E}\left(t_{A}\right)+\sum_{i} \gamma_{i} \cdot T_{i}^{L}\left(t_{A}\right) .
$$

The accompanying systematic utility function for estimation purposes becomes:

$$
V=\beta_{\tau} \cdot \tau+\sum_{i} \beta_{\alpha, i} \cdot T_{i}+\sum_{i} \beta_{\beta, i} \cdot T_{i}^{E}+\sum_{i} \beta_{\gamma, i} \cdot T_{i}^{L}
$$

After estimating the coefficients $\beta$, one can immediately determine $\alpha_{i}=\beta_{\alpha, i} / \beta_{i} ; \beta_{i}=\beta_{\beta, i} l \beta_{i}$; and $\gamma_{i}=\beta_{\gamma, i} / \beta_{\tau}$. As a final step, one could next calculate $H_{i}=\alpha_{i}$, and $W_{i}=\alpha_{i}+\beta_{i}$ for early arrivals and $W_{i}=\alpha_{i}+\gamma_{i}$ for late arrivals. Note that we assume intervals to be defined such that $t^{*}$ defines the boundary between two intervals.

\subsection{Data}

The data used were obtained in 2004 from an internet survey among Dutch commuters, who were selected on the criterion of experiencing at least 10 minutes of congestion, at least 3 days per week. The first part of the survey collected the information on the respondents' current commuting behaviour. The second part was a stated choice experiment, consisting of 11 choice profiles. Rather than choosing one of four alternatives, the respondents were asked to indicate how often they would choose each alternative, when considering a total of 10 trips for their morning commute. The dataset contains 1105 respondents, yielding 12265 choice observations.

The choice experiment was based on a fractional factorial design (orthogonal nonlinear main effects design), using 4 design levels for 13 of the attributes and 2 design levels for 2 of the attributes. To make the alternatives as realistic as possible, the actual values of attributes were based on each respondent's current travel behaviour. Each choice profile contained four alternatives. The first three alternatives involved a car trip, and the fourth one public transport. An alternative was characterized by the mode of transport; the trip length; the travel cost (composed of fuel costs and a road charge for the car, and a ticket for public transport); a departure time; a travel time interval and an implied arrival time interval (both with a uniform distribution); and a division of total travel time over uncongested and congested driving.

The dependent variable used in the present study is the choice proportion allocated to each of the four alternatives by individuals. Uncertainty in travel time is a separate attribute in this stated choice experiment, and we follow the 'expected utility maximization' approach of Noland and Small (1995) to incorporate the uncertainty variable. This means that we compute the 'expected' travel time and early/late arrival in different time intervals. In what follows, $T_{i}$, $T_{i}^{E}$ and $T_{i}^{L}$ thus refer to expected numbers of minutes in time interval $i$.

The choice experiment was not constructed specifically for estimating the above specified time-dependent model, which caused restrictions in the estimation of equation (10). A first restriction is that an individual's preferred arrival time is available only for a trip with 
a duration implied by the individual's free-flow (uncongested) travel time. This preferred arrival time $P A T$, and the accompanying preferred departure time $P D T$, define an interval in which the ideal arrival time $t^{*}$ should be located; compare Figure 1 . We will not attempt to actually estimate $t^{*}$, but instead test a few specifications in which $t^{*}$ is defined as a weighted average, with weights equal across individuals, of $P D T$ and $P A T$. We tested various weights between 0 and 1 , and the results appeared to be robust for various weights.

Table 1. The definition and descriptive statistics of the explanatory variables

\begin{tabular}{|c|c|c|c|c|c|}
\hline $\begin{array}{l}\text { Variable } \\
\text { notation }\end{array}$ & Definition & Mean & Std. & Min. & Max. \\
\hline $\mathrm{T}_{-1}$ & $\begin{array}{c}\text { The expected travel time spent during the interval } \\
\text { between } 0-15 \text { minutes before } t^{*}\end{array}$ & 10.19 & 6.28 & 0 & 15 \\
\hline $\mathrm{T}_{-2}$ & $\begin{array}{l}\text { The expected travel time spent during the interval } \\
\text { between } \mathbf{1 5 - 3 0} \text { minutes before } t^{*}\end{array}$ & 12.20 & 4.57 & 0 & 15 \\
\hline $\mathrm{T}_{-3}$ & $\begin{array}{l}\text { The expected travel time spent during the interval } \\
\text { between } \mathbf{3 0 - 6 0} \text { minutes before } t^{*}\end{array}$ & 24.12 & 10.34 & 0 & 30 \\
\hline $\mathrm{T}_{-4}$ & $\begin{array}{l}\text { The expected travel time spent during the interval } \\
\text { between } \mathbf{6 0 - 9 0} \text { minutes before } t^{*}\end{array}$ & 16.03 & 13.41 & 0 & 30 \\
\hline $\mathrm{T}_{-5}$ & $\begin{array}{l}\text { The expected travel time spent during the interval } \\
\text { between } \mathbf{9 0 - 1 2 0} \text { minutes before } t^{*}\end{array}$ & 8.18 & 12.09 & 0 & 30 \\
\hline $\mathrm{T}_{-6}$ & $\begin{array}{l}\text { The expected travel time spent during the interval } \\
\text { between } \mathbf{1 2 0 - 1 5 0} \text { minutes before } \mathrm{t}^{*}\end{array}$ & 3.62 & 8.96 & 0 & 30 \\
\hline $\mathrm{T}_{-7}$ & $\begin{array}{l}\text { The expected travel time during the interval } \\
\text { between 150-180 minutes before } t^{*}\end{array}$ & 1.52 & 6.07 & 0 & 30 \\
\hline $\mathrm{T}^{\mathrm{E}}$ & $\begin{array}{c}\text { The expected time spent at the destination during } \\
\text { the interval between } \mathbf{0 - 1 5} \text { minutes before } \mathrm{t}^{*}\end{array}$ & 4.57 & 6.24 & 0 & 15 \\
\hline $\mathrm{T}^{\mathrm{E}}-2$ & $\begin{array}{c}\text { The expected time spent at the destination during } \\
\text { the interval between } \mathbf{1 5 - 3 0} \text { minutes before } \mathrm{t}^{*}\end{array}$ & 1.95 & 3.81 & 0 & 14 \\
\hline $\mathrm{T}_{1}^{\mathrm{L}}$ & $\begin{array}{l}\text { The expected time not spent at the destination } \\
\text { during the interval between } \mathbf{0 - 1 5} \text { minutes after } \mathrm{t} *\end{array}$ & 7.56 & 7.30 & 0 & 15 \\
\hline $\mathrm{T}_{2}^{\mathrm{L}}$ & $\begin{array}{l}\text { The expected time not spent at the destination } \\
\text { during the interval between } \mathbf{1 5 - 3 0} \text { minutes after } \mathrm{t}^{*}\end{array}$ & 4.45 & 6.46 & 0 & 15 \\
\hline $\mathrm{T}_{3}^{\mathrm{L}}$ & $\begin{array}{l}\text { The expected time not spent at the destination } \\
\text { during the interval between } \mathbf{3 0 - 6 0} \text { minutes after } \mathrm{t}^{*}\end{array}$ & 1.85 & 3.70 & 0 & 27 \\
\hline Cost & Sum of fuel cost and toll (in Euros) & 3.54 & 3.67 & 0 & 33.7 \\
\hline Uncertainty & $\begin{array}{l}\text { The difference between maximum and minimum } \\
\text { possible travel times (in minutes) }\end{array}$ & 15.93 & 16.43 & 0 & 168 \\
\hline
\end{tabular}

A second restriction is that the design of the experiment does not provide sufficient variation in arrival times at work to allow for unrestricted estimation of all coefficients $\beta$ for all time 
periods of interest. Since there are hardly any observations of a departure time after $t^{*}$, there is a nearly perfect correlation between $T_{i}^{L}$ and $T_{i}$ in intervals after $t^{*}$. We can therefore only estimate the sum of $\alpha_{i}$ and $\gamma_{i}$ for these late intervals. To assure that there are sufficient observations in each time interval, we choose 30 minutes as the typical interval size. Still, since most arrivals are less than 30 minutes from $t^{*}$, we were able to use two smaller intervals of 15 minutes just before and after $t^{*}$. The notation, description and descriptive statistics of the variables are shown in Table 1 . The utility function we actually estimate is then given by:

$$
V=\beta_{\tau} \cdot \tau+\sum_{i=-7}^{-1} \beta_{\alpha, i} \cdot T_{i}+\sum_{i=-2}^{-1} \beta_{\beta, i} \cdot T_{i}^{E}+\sum_{i=1}^{3}\left(\beta_{\alpha, i}+\beta_{\gamma, i}\right) \cdot T_{i}^{L}
$$

\section{Estimation results}

The choice models we estimate in this section are based on the specification of equation (11). The objective is to see if the estimated coefficients vary over the different time intervals, and more importantly, whether the variation of the estimates follows the pattern illustrated in Figure 1. Since the experiment involves mode choice, we specify an alternative-specific constant $\left(\mathrm{ASC}_{\mathrm{PT}}\right)$ for public transport, to capture the effect of respondents' preference associated to that particular mode. Furthermore, we add the variable 'uncertainty' for car alternatives, defined as the width of the possible arrival time interval as shown to the respondents, to account for the additional disutility associated with travel time uncertainty (apart from the scheduling costs). The public transport alternative was presented as completely reliable in the experiment.

As discussed in previous section, we should expect an individual's $t^{*}$ to be somewhere between individual's preferred departure time $(P D T)$ and preferred arrival time $(P A T)$ for a free-flow travel time trip. Because $P D T$ and $P A T$ were asked in the questionnaire and $t^{*}$ wasn't, the latter should somehow be derived from the former two. We have estimated a series of models by varying the individuals' locations of $t^{*}$, relative to their PDT and PAT. The best model, pragmatically defined as the one yielding the highest log likelihood value, is the one with $t^{*}=0.2 * P D T+0.8^{*} P A T$. The choice models we summarize in Table 2 are based on that particular specification.

The estimation results are summarized in Table 2. Two models are presented: (1) a conventional multinomial logit (MNL) model, and (2) an mixed logit (ML) model that accommodates the correlation amongst choice sets drawn from the same individual. For model stability, cost parameters were treated as non-random in our mixed logit models (see also Revelt and Train, 1998; Bhat and Sardesai, 2006). The random parameters in the mixed logit model are assumed to follow a normal distribution. ${ }^{3}$

\footnotetext{
${ }^{3}$ A number of mixed logit models with uniform and triangular distributions were also estimated, and the results were similar to the models with normal distribution. The models with normal distribution yield higher loglikelihood values. We also estimated the models with log-normal distribution, but most of these hardly converged.
} 
Table 2. Estimation results

\begin{tabular}{|c|c|c|c|c|c|}
\hline \multirow{2}{*}{$\begin{array}{l}\text { Explanatory } \\
\text { variable }\end{array}$} & \multirow{2}{*}{$\begin{array}{l}\text { Refers to } \\
\text { valuation }\end{array}$} & \multicolumn{2}{|c|}{$\begin{array}{l}\text { Model } 1 \\
\text { MNL }\end{array}$} & \multicolumn{2}{|c|}{$\begin{array}{c}\text { Model } 2 \\
\text { ML }\end{array}$} \\
\hline & & Coeff. & t-stat. & Coeff. & t-stat. \\
\hline \multicolumn{6}{|c|}{ Random parameter mean effects } \\
\hline $\mathrm{T}_{-1}$ & $\alpha_{-1}$ & .0040 & 0.36 & .0082 & 0.55 \\
\hline $\mathrm{T}_{-2}$ & $\alpha_{-2}$ & .0002 & 0.05 & .0145 & 2.01 \\
\hline $\mathrm{T}_{-3}$ & $\alpha_{-3}$ & -.0107 & -6.19 & -.0172 & -4.26 \\
\hline $\mathrm{T}_{-4}$ & $\alpha_{-4}$ & -.0215 & -11.63 & -.0416 & -10.89 \\
\hline $\mathrm{T}_{-5}$ & $\alpha_{-5}$ & -.0269 & -10.24 & -.0471 & -9.78 \\
\hline $\mathrm{T}_{-6}$ & $\alpha_{-6}$ & -.0311 & -7.03 & -.0544 & -6.28 \\
\hline $\mathrm{T}_{-7}$ & $\alpha_{-7}$ & -.0414 & -6.22 & -.0592 & -3.69 \\
\hline $\mathrm{T}^{\mathrm{E}}{ }_{-1}$ & $\beta_{-1}$ & .0063 & 0.53 & .0137 & 0.81 \\
\hline $\mathrm{T}^{\mathrm{E}}{ }_{-2}$ & $\beta_{-2}$ & -.0370 & -5.14 & -.0244 & -1.92 \\
\hline $\mathrm{T}^{\mathrm{L}}{ }_{1}$ & $\alpha_{1}+\gamma_{1}$ & -.0173 & -4.42 & -.0220 & -3.51 \\
\hline $\mathrm{T}_{2}^{\mathrm{L}}$ & $\alpha_{2}+\gamma_{2}$ & -.0370 & -6.76 & -.0456 & -4.42 \\
\hline $\mathrm{T}_{3}^{\mathrm{L}}$ & $\alpha_{3}+\gamma_{3}$ & -.0299 & -3.88 & -.0576 & -3.91 \\
\hline Uncertainty & & -.0027 & -2.00 & -.0079 & -2.32 \\
\hline \multicolumn{6}{|c|}{ Random parameter standard deviation } \\
\hline $\mathrm{T}_{-1}$ & & & & .0075 & 0.64 \\
\hline $\mathrm{T}_{-2}$ & & & & .0086 & 13.91 \\
\hline $\mathrm{T}_{-3}$ & & & & .0087 & 23.79 \\
\hline $\mathrm{T}_{-4}$ & & & & .0064 & 19.45 \\
\hline $\mathrm{T}_{-5}$ & & & & .0046 & 10.17 \\
\hline $\mathrm{T}_{-6}$ & & & & .0048 & 7.35 \\
\hline $\mathrm{T}_{-7}$ & & & & .0095 & 6.40 \\
\hline $\mathrm{T}^{\mathrm{E}}{ }_{-1}$ & & & & .0032 & 0.35 \\
\hline $\mathrm{T}^{\mathrm{E}}{ }_{-2}$ & & & & .0043 & 0.21 \\
\hline $\mathrm{T}^{\mathrm{L}}{ }_{1}$ & & & & .0022 & 2.92 \\
\hline $\mathrm{T}^{\mathrm{L}}{ }_{2}$ & & & & .0088 & 12.35 \\
\hline $\mathrm{T}_{3}^{\mathrm{L}}$ & & & & .0058 & 3.84 \\
\hline Uncertainty & & & & .0060 & 28.29 \\
\hline \multicolumn{6}{|c|}{ Non-random parameters } \\
\hline Cost & & -.0989 & -17.18 & -.1957 & -34.77 \\
\hline ASC, PT & & -.9496 & -18.34 & -1.487 & -24.86 \\
\hline Observations $(\mathrm{N})$ & & \multicolumn{2}{|c|}{12265} & \multicolumn{2}{|c|}{12265} \\
\hline Log likelihood & & \multicolumn{2}{|c|}{-15401.38} & \multicolumn{2}{|c|}{-13141.79} \\
\hline R-sqrd Adjusted & & \multicolumn{2}{|c|}{0.0921} & \multicolumn{2}{|c|}{0.2265} \\
\hline
\end{tabular}

The main result is that the values of travel time savings $\alpha_{i}$ indeed are not constant over time, and the values increase as the time interval move further away from $t^{*}$. These results imply that individuals do have shadow prices that vary over time. The implied marginal rates of substitution between time attributes and money, i.e., the various shadow prices of interest, are shown in Table 3, and the patterns generally follow those hypothesized in Figure 1.

The design of the underlying questionnaire in the first place allows us to provide estimates of $\alpha_{i}$, and hence the function $H$, for all intervals before $t^{*}$. For example the MNL estimates of Model 1 depict how this value dramatically falls over time during the period prior to $t^{*}$, with the time coefficient becoming insignificantly different from zero for 
intervals -2 and -1 , reflecting that the individuals are indifferent between spending those minutes at home or in the car. This pattern thus replicates the hypothesized pattern in Figure 1, and suggests that the usual assumption that $\alpha$ is constant over time may be rather restrictive. We can estimate $\beta_{i}$ only for intervals -1 and -2 , and therefore the same holds for the determination of the function $W$ before $t^{*}$. For both models, the associated time coefficient is significantly different from zero only in interval -2 . Because $\beta_{-2}$ exceeds $\alpha_{-2}$, it appears that $W_{-2}$ is negative: during that time interval, people prefer to be in the car over being already at the destination. For intervals after $t^{*}$, we can only estimate the sum $\alpha_{i}+\gamma_{i}$, which is equal to $W_{i}$, and not the two components separately. (Because no separate estimate of $\alpha_{i}$ can be made, also $H_{i}$ can not be determined for intervals after $t^{*}$.) The pattern clearly shows a sharp increase from interval 1 to interval 3, which again confirms our hypotheses illustrated in Figure 1.

Table 3. Mean monetary values (Euro/hour)

\begin{tabular}{ccc}
\hline Monetary values & Model 1 & Model 2 \\
& MNL & ML \\
\hline$\alpha_{-1}$ & -2.43 & $-2.50(2.31)$ \\
$\alpha_{-2}$ & -0.13 & $-4.46(26.22)$ \\
$\alpha_{-3}$ & 6.48 & $5.28(26.54)$ \\
$\alpha_{-4}$ & 13.03 & $12.75(19.76)$ \\
$\alpha_{-5}$ & 16.35 & $14.44(14.17)$ \\
$\alpha_{-6}$ & 18.87 & $16.69(14.71)$ \\
$\alpha_{-7}$ & 25.15 & $18.16(29.05)$ \\
$\beta_{-1}$ & -3.84 & $-4.21(0.97)$ \\
$\beta_{-2}$ & 22.46 & $7.49(1.31)$ \\
$\alpha_{1}+\gamma_{1}$ & 10.47 & $6.75(6.72)$ \\
$\alpha_{2}+\gamma_{2}$ & 22.47 & $13.97(26.97)$ \\
$\alpha_{3}+\gamma_{3}$ & 18.12 & $17.67(17.93)$ \\
VUNC (uncertainty) & 1.65 & $2.43(18.54)$ \\
\hline Notes: 1. The derived monetary values from the standard deviation of random parameters are shown in \\
parenthesis; \\
2. Values or standard deviations arising from insignificant (at 90\% significance level) coefficients are in \\
italics (and in red in colour prints)
\end{tabular}

Model 2 is preferred to Model 1 since the former allows for taste heterogeneity across individuals as well as accommodating the correlations across choice sets that drawn from the same individuals (mixed logit with panel structure). The log-likelihood value also suggests that the model fit for Model 2 is much better than for Model 1. Although the mean monetary values differ somewhat between Models 1 and 2 (see Table 3), the two models seem to produce reasonable consistent results in terms of the qualitative patterns. It is interesting to note that the standard deviations of random parameters in Model 2 are mostly significant and large. It makes intuitive good sense that there is variation in parameters across individuals. Note that the negative $A S C_{P T}$ in both models suggests that our respondents have an inherent 
preference for car over public transport. This is consistent with the fact that our sampled respondents are all frequent car users.

Figure 3 summarizes the above findings graphically, by comparing the estimated timedependent patterns of $H$ and $W$ from the two time-dependent models ( 1 and 2) to the patterns that result from the conventional linear model estimated for the same data, for which we used estimates of $\alpha, \beta$ and $\gamma$ as reported in Tseng et al. (2005) for their model 2. The horizontal axis depicts time, where the ideal arrival time $t^{*}$ is set at $t=0$ for the time-dependent model, and the preferred arrival time $P A T$ for the linear model. The falling pattern of $H$ for times before $t^{*}$ is, by construction, of course not found in the linear model, but the time-independent value of $H$ seems to be reasonably close to the time average for the time-dependent models. The negative values for $W$ in interval -2 in the time-dependent models are consistent with a consistently negative value of $W$ before PAT in the linear model ( $\beta>\alpha$ in that model). Apparently, there is a tendency among the respondents to be keen on avoiding spending time at work before the start time: time spent in the car is valued higher than time spent at work in our estimates. For times after $t^{*}$, the linear model implies that $H$ maintains its pre- $t^{*}$ value; there are insufficient observations to estimate the corresponding values for the timedependent model, but the last value estimated, not significantly different from zero, suggests that lower values would have resulted, just as hypothesized in Figure 1. Finally, $W$ rises as the time interval moves forward, but stays below the value implied by the linear model. The biggest difference between the time-dependent models versus the linear model therefore seems to be that the latter cannot reproduce the falling pattern of $H$, corresponding to the conventional value of travel time savings $\alpha$, over the period prior to the ideal arrival time $t^{*}$. Comparing the two time-dependent models, the various estimates seem reasonably close, with the mixed logit model producing less extreme values and smoother patterns than its multinomial counterpart.

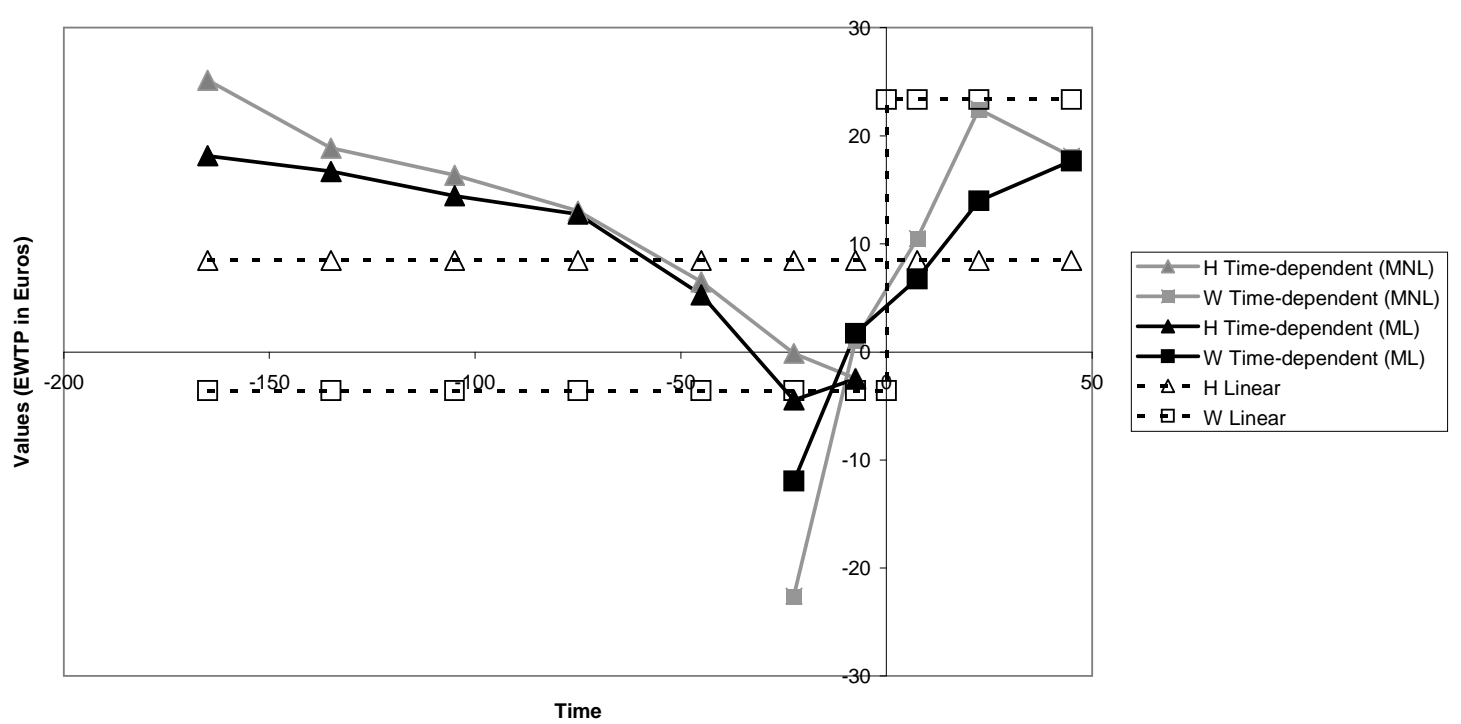

Figure 3. The empirical EWTP-functions for the time-dependent and the linear models 
There is one final potential caveat to be addressed. Because the design of the stated choice experiment was such that arrival times were varied around the most desired arrival time, we have relatively many observations for respondents making longer trips in the earlier intervals. Now if for some reason these individuals have a higher value of travel time savings, the patterns depicted for $H$ in Figure 3 might also be due to a different sampling of respondents along the time axis. We have therefore also estimated the same time-dependent models for the $48 \%$ of respondents making the longest trips $(>40 \mathrm{~km})$. The results conveyed the same qualitative patterns as those shown in Figure $3 .{ }^{4}$

We also verified whether the results vary by socio-economic characteristics. Estimation results, reworked into monetary values as in Table 3, are provided in the Appendix. A separation by income into two groups left the qualitative patterns, as shown in Figure 3, intact. It further revealed that the higher income group has higher $\alpha$ s before $t^{*}$, whereas the $\beta$ s for arrivals before $t^{*}$, and $\alpha+\gamma$ for arrivals after $t^{*}$, are higher for lower income groups. This confirms the expectation that higher income groups are willing to pay more to avoid travel time, whereas lower income groups have tighter scheduling constraints. A separation by gender produces somewhat less clear-cut results, partly because there are too few observations for female drivers in some time periods. But when they travel, women appear to usually have higher values than male drivers, for the shadow prices listed in Table 3. And finally, we tested whether the inclusion of a lateness penalty (a probability in our model, because we have an uncertain travel time), also introduced by Small (1982), affects the results. The coefficient for this variable turned out to be statistically insignificant, both in MNL and in ML estimations, and the other coefficients are (not surprisingly) hardly affected.

\section{Conclusion}

We proposed an alternative, dynamic framework for estimating time-varying values of travel time savings and values of schedule delay. Our formulation represents time-preferences as the time-varying excess willingness to pay (EWPT) to being in the one location, over being elsewhere. We applied the framework to SP data representing the respondents' departure time choices for the morning commute. We showed how the conventional linear model is a special case of our model, and that the conventional model is implausible particularly in that it implicitly assumes that the willingness to pay for spending a minute at home instead of being in the vehicle does not vary by time of day, even not for very early departures. It is especially in this respect that the estimates for the time-dependent model deviate substantially from those for the stationary model, estimated for the same data. The data thus support the case for our time-dependent framework rather convincingly.

\footnotetext{
${ }^{4}$ For this estimate, we had to merge the two 15 minutes intervals prior to $t^{*}$ to one 30 minute interval. For the MNL model, the values of $H$ were as follows: $H_{-1 \& 2}=-7.0$ (insign.); $H_{-3}=1.3$ (insign.); $H_{-4}=15.4 ; H_{-5}=20.0$; $H_{-6}=20.5 ; H_{-7}=29.0 ;$ and the values for $W$ were as follows: $W_{1}=5.6$ (insign.); $W_{2}=25.3 ; W_{3}=27.1$. For the ML model, the values of $H$ were as follows: $H_{-1 \& 2}=-1.8$ (insign.); $H_{-3}=-2.4$ (insign.); $H_{-4}=12.9 ; H_{-5}=20.0 ; H_{-}$ ${ }_{6}=19.5 ; H_{-7}=23.3$; and the values for $W$ were as follows: $W_{1}=7.2 ; W_{2}=11.1 ; W_{3}=20.0$.
} 
Because the conventional linear model is a special, constrained case of the timedependent model that we propose, it seems that there is little point in discussing which model is preferable from a theoretical or a behavioural viewpoint. That is, if the data allow, it seems preferable to estimate the time-dependent model and deciding next whether imposing the conventional constraint of a constant value of travel time savings $\alpha$ seems justifiable. From a practical perspective, clearly, there may be other considerations. If anything, a proper estimation of the time-dependent model requires a rather rich data set, with wide ranges of departure and arrival times, in particular if the EWPT for being at work is to be estimated also for intervals before the ideal arrival time $t^{*}$, and the EWPT for being at home is to be estimated also for intervals after $t^{*}$.

Our results suggest that individuals' time-related shadow prices vary strongly over the morning peak, and values of travel time savings are consequently strongly time-dependent. A failure to incorporate such considerations may produce biased estimates of values of travel time savings, and errors in the prediction of behavioural responses to policies or other measures that affect the time pattern of congestion in the morning peak. This, in turn, may of course also affect the accuracy of cost-benefit calculations for such measures. It seems difficult to predict, in general, the relative size and sign of such biases. Studying this question in the context of a dynamic equilibrium model would be an interesting topic for further study.

\section{References}

Arnott, R., A. de Palma and R. Lindsey (1993) "A structural model of peak- period congestion: a traffic bottleneck with elastic demand" American Economic Review 83 (1) 161-179.

Ashiru, O., J.W. Polak, and R.B. Noland (2004) "The utility of schedules: A theoretical model of departure time choice and activity time allocation with application to individual activity schedules" Transportation Research Record 1894 84-98.

Bates, J.J. (1987) "Measuring travel time values with a discrete choice model: a note" Economic Journal 97 493-498.

Becker, G.S. (1965) "A theory of the allocation of time" Economic Journal 75 493-517.

Bhat, C. R. and R. Sardesai (2006) "The impact of stop-making and travel time reliability on commute mode choice" Transportation Research 40B 709-730.

DeSerpa, A.C. (1971) "A theory of the economics of time" Economic Journal 81 828-846.

Ettema, D.F., H.J.P. Timmermans (2003) "Modeling departure time choice in the context of activity scheduling behaviour" Transportation Research Record 1831 39-46.

Johnson, M. (1966) "Travel time and the price of leisure" Western Economic Journal Spring 135-145.

Liu, H.X., X. He and W. Recker (2007) "Estimation of time-dependency of values of travel time and its reliability from loop detector data" Transportation Research 41B 448-461.

Noland, R.B. and K.A. Small, (1995) "Travel-time uncertainty, departure time choice, and the cost of morning commutes" Transportation Research Record 1493 150-158.

Oort, C.J. (1969) "The evaluation of travelling time" Journal of Transport Economics and Policy 3 279-286.

Redmond, L.S. and P.L. Mokhtarian (2001) "The positive utility of the commute: modeling ideal commute time and relative desired commute amount" Transportation 28 179-205.

Revelt, D. and K. Train (1998) "Mixed logit with repeated choices: households' choices of appliance efficiency level" The Review of Economics and Statistics 80 (4) 647-657.

Small, K.A. (1982) "The scheduling of consumer activities: work trips" American Economic Review 72 (3) $467-479$.

Small, K.A., C. Winston and J. Yan (2005) "Uncovering the distribution of motorists' preferences for travel time and reliability" Econometrica 73 1367-1382. 
Tseng, Y., B. Ubbels, and E.T. Verhoef (2005) "Value of time, schedule delay and reliability estimation based on choice behaviour of Dutch commuters facing congestion". Paper presented at the $45^{\text {th }}$ ERSA congress, VU University Amsterdam.

Van Amelsfort, D. and M. Bliemer (2005) "Valuation of uncertainty in travel time and arrival time some findings from a choice experiment". Paper presented at the $45^{\text {th }}$ ERSA congress, VU University Amsterdam.

Vickrey, W.S. (1969) "Congestion theory and transport investment" American Economic Review (Papers and Proceedings) 59 251-260. 


\section{Appendix Monetary values in Table 3 disaggregated by income and gender}

Monetary values by income and gender (MNL)

\begin{tabular}{ccccc}
\hline \multirow{2}{*}{ Monetary values } & \multicolumn{2}{c}{ Income } & \multicolumn{2}{c}{ Gender } \\
\cline { 2 - 5 } & Low & High & Male & Female \\
& MNL & MNL & MNL & MNL \\
\hline$\alpha_{-1}$ & -9.29 & 4.46 & -4.11 & 2.39 \\
$\alpha_{-2}$ & -5.53 & 3.47 & 1.33 & -3.46 \\
$\alpha_{-3}$ & 3.44 & 8.18 & 3.84 & 12.72 \\
$\alpha_{-4}$ & 12.63 & 13.16 & 13.10 & 13.95 \\
$\alpha_{-5}$ & 19.45 & 14.06 & 15.29 & 23.68 \\
$\alpha_{-6}$ & 17.94 & 21.73 & 19.65 & 16.38 \\
$\alpha_{-7}$ & - & 31.92 & 26.69 & - \\
$\beta_{-1}$ & -8.32 & 1.97 & -5.71 & 0.15 \\
$\beta_{-2}$ & 34.95 & 13.38 & 22.54 & 18.08 \\
$\alpha_{1}+\gamma_{1}$ & 12.20 & 8.97 & 11.17 & 7.39 \\
$\alpha_{2}+\gamma_{2}$ & 37.51 & 13.74 & 18.88 & 36.17 \\
$\alpha_{3}+\gamma_{3}$ & - & 24.35 & 21.48 & - \\
VUNC (uncertainty) & 2.25 & 1.11 & 2.69 & -1.66 \\
\hline
\end{tabular}

Monetary values by income and gender (ML)

\begin{tabular}{ccccc}
\hline \multirow{2}{*}{ Monetary values } & \multicolumn{2}{c}{ Income } & \multicolumn{2}{c}{ Gender } \\
\cline { 2 - 5 } & Low & High & Male & Female \\
& ML & ML & ML & ML \\
\hline$\alpha_{-1}$ & -8.36 & 4.37 & -4.74 & 0.61 \\
$\alpha_{-2}$ & -11.88 & 0.35 & -4.25 & -6.98 \\
$\alpha_{-3}$ & 2.35 & 8.22 & 2.06 & 9.64 \\
$\alpha_{-4}$ & 13.79 & 12.51 & 11.35 & 13.84 \\
$\alpha_{-5}$ & 15.23 & 13.98 & 14.41 & 21.23 \\
$\alpha_{-6}$ & 16.50 & 18.37 & 17.16 & 10.42 \\
$\alpha_{-7}$ & - & 32.76 & 33.43 & - \\
$\beta_{-1}$ & -9.35 & 2.00 & -6.75 & 0.32 \\
$\beta_{-2}$ & 14.61 & 3.69 & 8.02 & 5.54 \\
$\alpha_{1}+\gamma_{1}$ & 6.18 & -6.83 & 7.69 & 3.01 \\
$\alpha_{2}+\gamma_{2}$ & 24.90 & 9.43 & 11.90 & 23.83 \\
$\alpha_{3}+\gamma_{3}$ & - & 18.47 & 17.18 & - \\
VUNC (uncertainty) & 4.51 & 1.00 & 2.98 & 2.64 \\
\hline
\end{tabular}

Note: Low incomes are defined as yearly gross household incomes of less than $€ 45000$. 